\section{Vol. 30, Issue 5, May 2007}

Editorial

226 Chemotherapy for Osteosarcoma without High-Dose Methotrexate: Another Piece in the Puzzle Blay, J.-Y, (Lyon)

Original Articles

228 Chemotherapy for Osteosarcoma without HighDose Methotrexate: A 12-Year Follow-Up on 53 Patients Tunn, P.U.; Reichardt, P. (Berlin)

235 Mitomycin C plus Infusional 5-Fluorouracil in Platinum-Refractory Gastric Adenocarcinoma: An Extended Multicenter Phase II Study

Hartmann, J.T.; Pintoffl, J.P. (Tübingen); Al-Batran, S.E. (Frankfurt/M.); Quietzsch, D. (Chemnitz); Meisinger, I.; Horger, M.; Nehls, O.; Bokemeyer, C.; Königsrainer, A. (Tübingen); Jäger, E. (Frankfurt/M.); Kanz, L. (Tübingen)

243 Diagnostic Value of Ductoscopy in the Diagnosis of Nipple Discharge and Intraductal Proliferations in Comparison to Standard Methods Grunwald, S.; Heyer, H. (Greifswald); Paepke, S. (München); Schwesinger, G.; Schimming, A. (Greifswald); Hahn, M. (Tübingen); Thomas, A. (Berlin); Jacobs, V.R. (München); Ohlinger, R. (Greifswald)

Clinical Cases

249 Penile Metastasis from a T1b Prostate Carcinoma Cai, T.; Salvadori, A.; Nesi, G.; Detti, B.; Tinacci, G.; Zini, E.; Bartoletti, R. (Florence)

253 Atrial Fibrillation Induced by Gemcitabine Treatment in a 65-Year-Old Man Tavil, Y.; Arslan, U.; Okyay, K.; Sen, N.; Boyacı, B. (Ankara)

256 Multiple Littoral Cell Angioma of the Spleen: A Case Report and Review of the Literature Qu, Z.B.; Liu, L.X.; Wu, L.F.; Zhao, S.; Jiang, H.C. (Harbin)

\section{Review Articles}

260 Do the Results of the New Trials Change the Standard Treatment of Metastatic Renal Cell Cancer?

Mulder, S.F.; van Spronsen, D.J.; De Mulder, P.H.M. (Nijmegen)
Band 30, Heft 5, Mai 2007

Editorial

226 Chemotherapie ohne hoch dosiertes Methotrexat beim Osteosarkom: Ein weiterer Puzzlestein Blay, J.-Y. (Lyon)

Originalarbeiten

228 Behandlungsergebnisse ohne hoch dosiertes Methotrexat beim Osteosarkom: Eine retrospektive 12-Jahres-Studie bei 53 Patienten

Tunn, P.U.; Reichardt, P. (Berlin)

235 Mitomycin C plus intravenöses 5-Fluorouracil bei platinrefraktären Adenokarzinomen des Magens: Eine erweiterte multizentrische Phase-II-Studie Hartmann, J.T.; Pintoffl, J.P. (Tübingen); Al-Batran, S.E. (Frankfurt/M.); Quietzsch, D. (Chemnitz); Meisinger, I.; Horger, M.; Nehls, O.; Bokemeyer, C.; Königsrainer, A. (Tübingen); Jäger, E. (Frankfurt/M.); Kanz, L. (Tübingen)

243 Stellenwert der Duktoskopie bei unklarer Mamillensekretion und intraduktalen Proliferationen im Vergleich mit Standardverfahren Grunwald, S.; Heyer, H. (Greifswald); Paepke, S. (München); Schwesinger, G.; Schimming, A. (Greifswald); Hahn, M. (Tübingen); Thomas, A. (Berlin); Jacobs, V.R. (München); Ohlinger, R. (Greifswald)

Kasuistiken

249 Penismetastase eines T1b-Prostatakarzinoms Cai, T.; Salvadori, A.; Nesi, G.; Detti, B.; Tinacci, G.; Zini, E.; Bartoletti, R. (Florence)

253 Vorhofflimmern unter Gemcitabintherapie bei einem 65-jährigen Patienten

Tavil, Y.; Arslan, U.; Okyay, K.; Sen, N.; Boyacı, B. (Ankara)

256 Multiples Uferzellangiom der Milz: Ein Fallbericht und Übersicht über die Literatur Qu, Z.B.; Liu, L.X.; Wu, L.F.; Zhao, S.; Jiang, H.C. (Harbin)

Übersichtsarbeiten

260 Haben die Ergebnisse neuer Studien einen Einfluss auf die Standardtherapie des metastatischen Nierenzellkarzinoms? Mulder, S.F.; van Spronsen, D.J.; De Mulder, P.H.M. (Nijmegen)

\begin{tabular}{ll}
\hline KARGER & $\oplus$ 2007 S. Karger GmbH, Freiburg \\
Fax +49761 4520714 & Artikel (Volltext) und Inhaltsverzeichnisse \\
$\begin{array}{l}\text { E-mail Information@Karger.de } \\
\text { www.karger.com }\end{array}$ & $\begin{array}{l}\text { sowie das vorläufige Inhaltsverzeichnis des nächsten Heftes: } \\
\text { www.karger.com/onk_bk.htm }\end{array}$
\end{tabular}




\section{Vol. 30, Issue 5, May 2007}

265 Report on the 1st International Workshop of the German Competence Network Malignant Lymphomas Re, D.; Elter, T.; Hallek, M. (Köln)

Reports of Oncological Societies

274 Chirurgische Arbeitsgemeinschaft Onkologie (CAO-V) der Deutschen Gesellschaft für Viszeralchirurgie

276 PharmaNews / PharmaTicker

279 Imprint

280 Meetings and Conferences

282 Guidelines for Authors

Forthcoming papers are listed on page 284 .

\section{Band 30, Heft 5, Mai 2007}

265 Bericht über den ersten Internationalen Workshop des deutschen Kompetenznetzes Maligne

Lymphome (KML)

Re, D.; Elter, T.; Hallek, M. (Köln)

Mitteilungen onkologischer Gesellschaften

274 Chirurgische Arbeitsgemeinschaft Onkologie (CAO-V) der Deutschen Gesellschaft für Viszeralchirurgie

276 PharmaNews / PharmaTicker

279 Impressum

280 Tagungen und Kongresse

283 Hinweise für Autoren

Einen Ausblick auf den Inhalt der kommenden Hefte finden Sie auf Seite 284.

\section{KARGER}

Fax +497614520714

E-mail Information@Karger.de

www.karger.com
() 2007 S. Karger GmbH, Freiburg

Artikel (Volltext) und Inhaltsverzeichnisse

sowie das vorläufige Inhaltsverzeichnis des nächsten Heftes: www.karger.com/onk_bk.htm 